\title{
Characterizing Ultrafine Grained Material using EBSD
}

\author{
Andrew Deal, Radhakrishna Bhat, Richard DiDomizio, Judson Marte, PR Subramanian
}

GE Global Research, One Research Circle, Niskayuna, NY 12309

Ultrafine grained (UFG) alloys, those with grain sizes significantly below 1 micron, are of general interest from both a property and processing standpoint. Improved mechanical properties have been reported, and many materials are under investigation $[1,2]$. The nanoscale nature of these materials, however, provides a significant number of characterization challenges. Here we discuss the use of Electron Backscatter Diffraction (EBSD) to characterize the grain size of ultrafine grained Ti-6Al-4V (UFG Ti64).

UFG Ti64 was produced by near-isothermal Multi-axis Forging (MAF) [3]. Samples of this material were heat treated to study the static coarsening of the alpha phase, and hot compression tests were performed on UFG Ti64specimens to examine dynamic coarsening behavior. After heat treatment or compression, samples were metallographically prepared in conductive 1.25 inch mounts according to a modified version of a published technique [4]. To understand the coarsening kinetics, it was essential to quantify the average alpha grain size of the material. A high spatial resolution and ability to examine large areas for statistical purposes were critical requirements for this task, making a FEG-SEM with EBSD the instrumentation of choice.

Since large EBSD measurements are on the order of hours, even with modern camera speeds, maintaining mechanical and thermal stability of the SEM was critical. Accordingly, the FEGSEM used for UFG grain size measurements was enclosed to isolate it from thermal oscillations inherent to HVAC systems. A chiller with precise PID temperature control was installed to keep the lens cooling stable. These modifications helped keep the short-term temperature fluctuations below $0.5^{\circ} \mathrm{C}$. To minimize long term mechanical or thermal drift, the sample was positioned for analysis under appropriate beam conditions and then rested for a minimum of 3 hours prior to the EBSD measurements. Long term temperature drift was typically less than $1{ }^{\circ} \mathrm{C}$.

Figure 1 shows the band contrast for a small region of tested UFG Ti64 material. Small maps such as these were used to examine the resolution of the EBSD measurements and get a sense of the grain size. Reasonable grain size statistics, however, were accumulated through EBSD line scans along the tilt axis. For each measurement, the step size within each line was $20 \mathrm{~nm}$, and lines were spaced between 1 and 50 microns apart. Scan times were on the order of 14 hours.

Initial results of the static coarsening experiments are shown in Figure 2, compared with conventional Ti64 material analyzed optically. The EBSD measurements show that the alpha grain size of the UFG Ti64 is reasonably stable with respect to static coarsening at $650^{\circ} \mathrm{C}$. It remained submicron after an hour and remained below 3 microns after 77 hours.

\section{References}

[1] S. Han, et al. Metallurgical and Materials Transactions A 36 (2005) 467-470.

[2] W.J. Kim, et al. Metallurgical and Materials Transactions A 33 (2002) 3155-3164.

[3] R. DiDomizio, et al. Materials Science Forum 503-504 (2006) 793-798.

[4] S. Di Iorio, et al. Acta Materialia 55 (2007) 105-118.

Acknowledgement - This work is supported in part by NIST under grant 70NANB7H7038, in which GE is a joint investigator with ATI Allvac. 


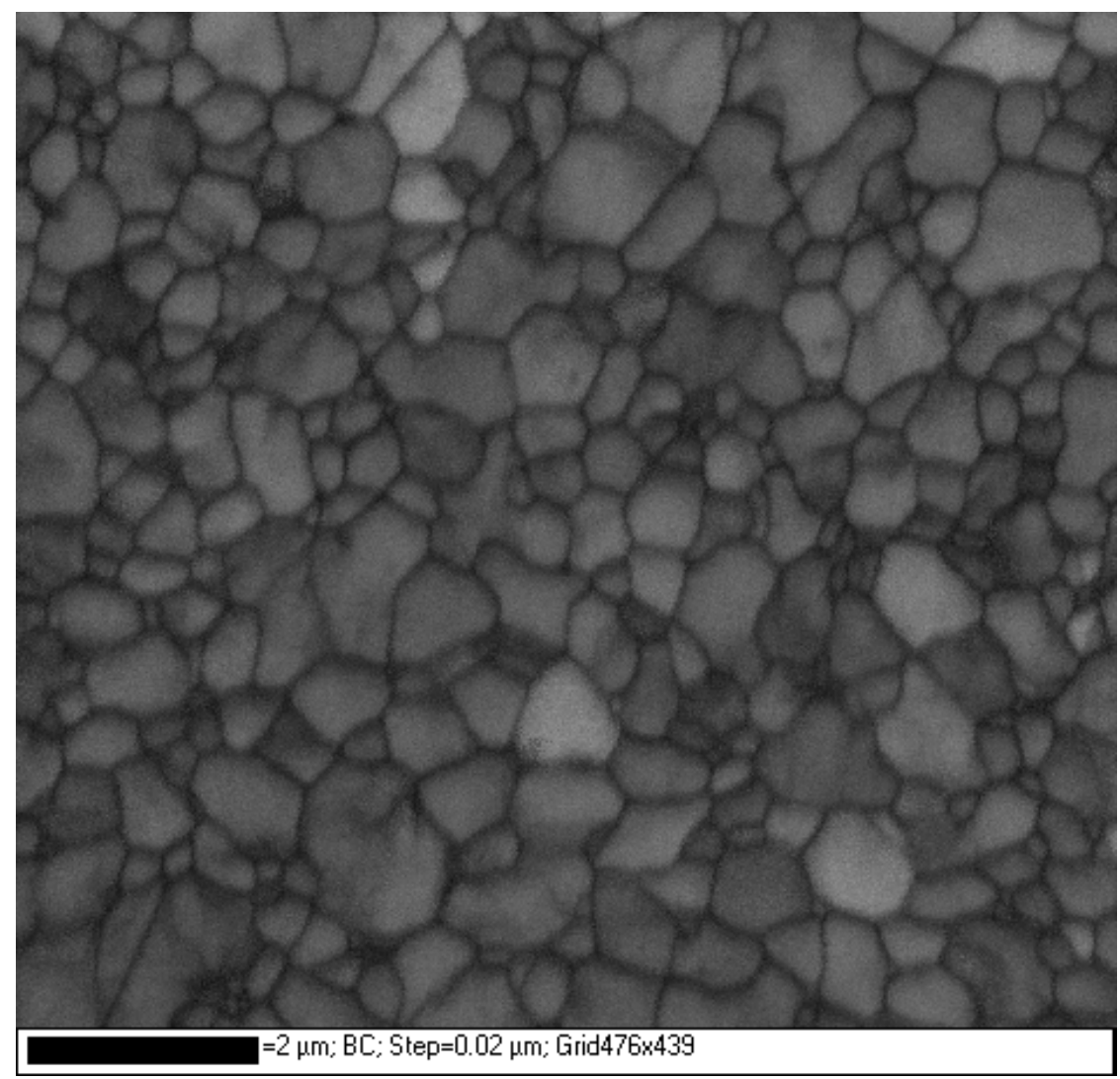

Fig. 1 - Band contrast EBSD map of UFG Ti64 exposed to $537^{\circ} \mathrm{C}$ for 77 hours.

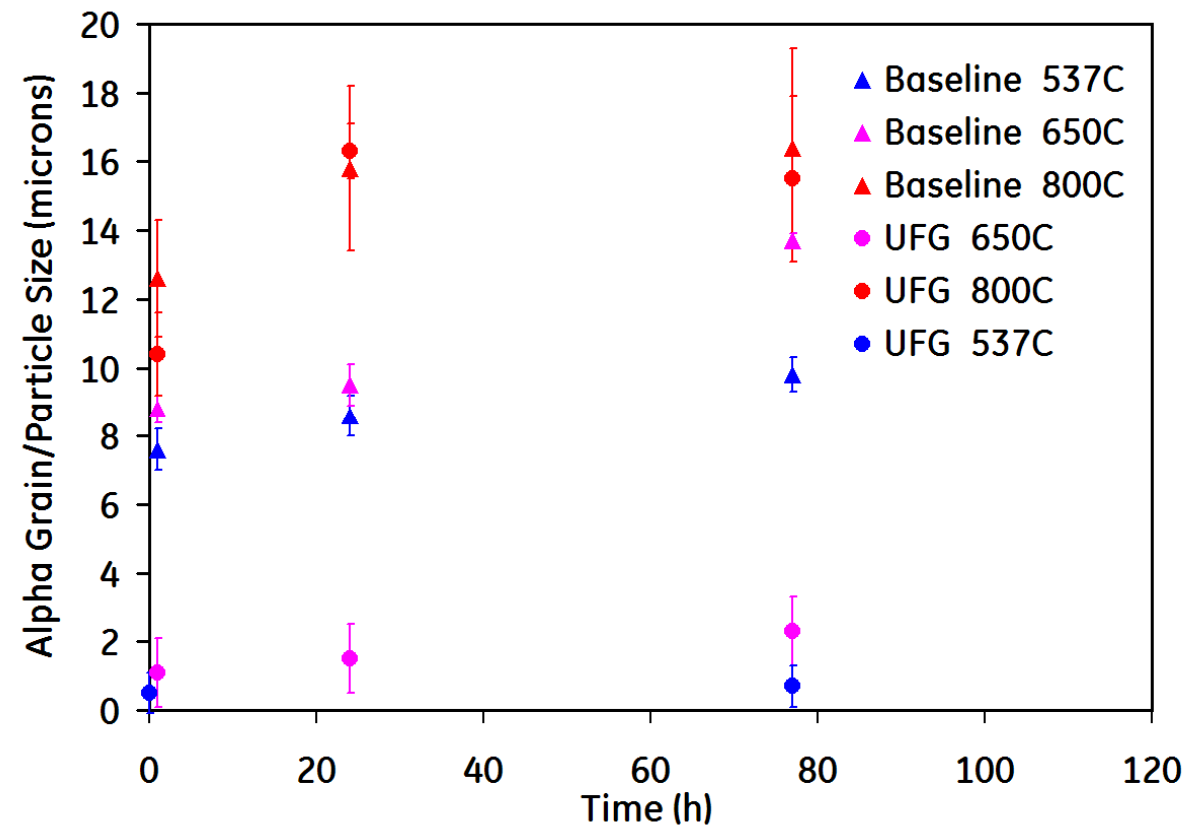

Fig. 2 - Alpha grain size measurements from conventional and UFG Ti64 as a function of time at temperature. Measurements below 5 microns were made using EBSD. 